\title{
FICAR OU PARTIR? \\ Um estudo sobre os consequentes de atitudes de vingança no contexto de serviços
}

\section{1- Simony Rodrigues Marins*}

Mestre em Administração pela Universidade Estadual do Ceará (PPGA/UECE), Brasil.

simonymarins@gmail.com

http://lattes.cnpq.br/7822187597252616

\section{2- Ana Paula Benício da Rocha}

Mestre em Administração pela Universidade Estadual do Ceará (PPGA/UECE), Brasil.

anapaulabenicio@hotmail.com

http://lattes.cnpq.br/2185182169756401

\section{3- Márcio de Oliveira Mota}

Doutor em Administração pela UNIFOR, Brasil.

Professor do Programa de Pós-Graduação em Administração da Universidade Estadual do Ceará (PPGA/UECE), Brasil. marcio@marciomota.com

http://buscatextual.cnpq.br/buscatextual/visualizacv.do?id=K4716139H6

\section{Editor responsável pela submissão:}

Diego Maganhotto Coraiola.

Artigo analisado via processo de revisão duplo cego (Double-blind).

Recebido em: 29/09/2014

Aprovado em: 20/05/2016

última Alteração: 19/06/2016

\footnotetext{
* Contato Principal: Rua da Paz, 215, 303. Mucuripe, Fortaleza - CE, Brasil. CEP: 60165-180
} 


\title{
FICAR OU PARTIR? UM ESTUDO SOBRE OS CONSEQUENTES DE ATITUDES DE VINGANÇA
} NO CONTEXTO DE SERVIÇOS

\begin{abstract}
RESUMO
Este artigo buscou analisar os consequentes de atitudes vingativas de consumidores no contexto de serviços. Para tanto foi feito um estudo descritivo de natureza quantitativa por meio de uma survey interseccional. Os dados foram analisados pela técnica da Modelagem de Equações Estruturais (MEE). Dentre os principais resultados conclui-se a relação positiva entre o desejo de vingança e atitudes vingativas, e atitudes vingativas com o desejo de afastamento. Não foi significativa a relação entre atitudes vingativas e o desejo de reconciliação e não houve, além, efeito mediador entre o desejo de vingança e o desejo de afastamento.
\end{abstract}

\section{Palavras-Chave}

Consequentes; Atitudes de vingança; Serviços.

\section{STAY OR LEAVE? A STUDY ABOUT THE CONSEQUENCES OF VENGEANCE'S BEHAVIORS IN SERVICES CONTEXT}

\section{ABSTRACT}

This article aimed to analyze the consequences of vengeful consumer behaviors in the context of services. For this purpose, a descriptive quantitative study was carried out by an intersectional survey. The data were analyzed using the Structural Equation Modeling (SEM). It provides empirical support for the positive relationship between the desire for revenge and vindictive behaviors and vindictive behaviors with the desire for avoidance. There was no significant relationship between vindictive behaviors and the desire for reconciliation and, there was not further mediating effect between the desire for revenge and the desire for avoidance.

\section{Keywords}

Consequences; Vindictive behaviors; Services. 


\section{Introdução}

A literatura registra várias reações apresentadas pelos consumidores durante as relações de troca de produtos e/ou serviços, quando conduzidas de forma malsucedida, influenciadas pela insatisfação, percepção de injustiça, raiva e/ou desejo de vingança em menor ou em maior grau, presentes, por exemplo, nos estudos de Bechwati e Morrin (2003), Bougie, Pieters e Zeelenberg (2003). Funches, Markley e Davis (2009), Grégoire, Laufer, Tripp e Legoux (2009), Hopkins (2013), Huefner e Hunt (2000), Huefner et al. (2002), Joireman, Grégoire, Devezer e Tripp (2013), Lee, Pan e Tsai (2013) e Thelen e Shapiro (2012). Essas emoções negativas originadas durante experiências de consumo malsucedidas têm desencadeado a prática de ações cada vez mais danosas à imagem das empresas, amplificadas pela acessibilidade fornecida pela Internet, que possibilita a disseminação de qualquer conteúdo para um grupo maior de pessoas (e.g. boca a boca negativo) (Ward \& Ostrom, 2006). Tais ações podem ser transmitidas em ambientes específicos ou não, como as redes sociais, o que oferece aos consumidores maior sensação de empoderamento (Albuquerque, Pereira, \& Belini, 2011; Andrade \& Brandão, 2012; Andrade, Balassiano, \& Brandão, 2013).

Diversos autores apontam a necessidade de investigação de aspectos negativos do comportamento do consumidor, enfatizando a vingança como ação relativamente pouco estudada, ainda que consista em importante fenômeno comportamental (Bechwati \& Morrin, 2003; Cronin, 2003; Funches et al., 2009). Nesse contexto, considera-se de extrema relevância o desenvolvimento de estudos que explorem os diversos aspectos desse fenômeno na atuação de consumidores insatisfeitos, a fim de oferecer às empresas oportunidade de melhoria nos processos de trocas comerciais e em suas estruturas de relacionamento com os clientes. Consumidores também serão diretamente beneficiados com os resultados de pesquisas dessa natureza, visto que a melhor compreensão de seus pensamentos, sentimentos e ações pode melhorar a oferta de produtos e serviços pelas empresas, reduzindo, consequentemente, a possibilidade de manifestação de comportamentos vingativos.

Na última década, embora tenha ocorrido crescimento desses estudos, principalmente em campo internacional, poucos autores no Brasil têm se empenhado em identificar os fatores que interferem em processos de vingança. Predominam, em seus estudos, objetos que evidenciam as razões originadoras e as formas como atitudes vingativas se manifestam, notadamente em ambientes virtuais de compra. Considera-se, assim, necessário o aprofundamento da compreensão do comportamento vingativo de consumidores em situações de contato direto com a empresa (on stage) e não somente virtual, de modo particular com empresas prestadoras de serviços, além do estudo dos fatores que compõem esse fenômeno e seus consequentes (Albuquerque et al., 2011; Andrade \& Brandão, 2012; Andrade, Brandão, Pereira, \& Cunha, 2010; Andrade et al., 2013; Fernandes \& Santos, 2008; Giglio \& Chauvel, 2002; Santos \& Fernandes, 2010).

De maneira específica, registra-se a existência de poucos estudos, como os de Hopkins (2013) e Joireman et al. (2013), destinados a evidenciar os consequentes de atitudes vingativas de consumidores no contexto internacional. No Brasil, porém, ainda não há estudos dessa natureza, lacuna que levou à emergência da seguinte questão: Quais os consequentes de atitudes de vingança de consumidores no contexto da prestação de serviços?

Com o intuito de produzir respostas a esse questionamento, desenvolveu-se esta pesquisa, cujo objetivo geral consiste em analisar os consequentes de Atitudes Vingativas no contexto de serviços. Como objetivos específicos, destina-se a análise de quatro hipóteses balizadoras do modelo proposto:

- Hipótese 1: O Desejo de Vingança (DV) influencia positivamente Atitudes de Vingança (AV), como as Reclamações Vingativas, doravante denominada H1.

- Hipótese 2: Atitudes Vingativas (AV) influenciam positivamente o Desejo de Afastamento (DA), doravante denominada $\mathrm{H} 2$.

- Hipótese 3: Atitudes Vingativas (AV) como as Reclamações Vingativas (RV) não são mediadoras entre Desejo de Vingança (DV) e Atitudes de Afastamento (AF), doravante denominada $\mathrm{H} 3$.

- Hipótese 4: Há relação positiva entre Atitudes de Vingança (AV) e Desejo de Reconciliação (DR), doravante denominada H4.

No intuito de atingir o objetivo proposto desenvolveu-se um estudo descritivo com técnicas quantitativas, mediado pela realização de um levantamento (survey) interseccional, no qual se analisaram as relações entre os construtos de Desejo de Vingança (DV), Atitudes Vingativas (AV), Desejo de Afastamento (DA) e Desejo de Reconciliação (DR) a partir de um estudo com a técnica da Modelagem de Equações Estruturais (MEE). Buscou-se, ainda, desvendar a natureza dessas relações, 
lançar luz sobre o entendimento desse tema na tentativa de esclarecer tais questões e incentivar pesquisas futuras na área.

A seguir evidencia-se a literatura sobre as temáticas de vingança, alguns antecedentes e possíveis consequentes, os aspectos metodológicos, os resultados da pesquisa e, ao final, as respectivas discussões e considerações.

\section{Referencial Teórico}

\subsection{Alguns Antecedentes de Vingança}

A psicologia aborda a vingança como a inflição de um mal em troca da percepção de um dano sofrido, cujo objetivo principal é aliviar o vingador de um sentimento desconfortável (como a raiva). Não é necessariamente racional e pode incidentemente ocasionar agressão futura, de forma direta ou indireta, mensurada em atitudes vingativas (Stuckless \& Goranson, 1992).

Zourrig, Chebat e Toffoli (2009) afirmam que as emoções negativas são observadas como o principal catalisador de Atitudes Vingativas. A raiva é estudada como uma resposta emocional negativa mais geral que pode ter diversos destinos e muitos modos possíveis de expressão (Stuckless, Ford, \& Vitelli, 1995).

Funches (2011) a conceitua como um estado emocional que decorre da perda de percepção do consumidor de direito devido a uma experiência de consumo injusta, ameaçadora ou prejudicial. Essas experiências de consumo envolvem interações com a empresa, seus produtos, serviços e funcionários. A raiva pode ser subdividida em três categorias de principais causadores: promessas quebradas (serviço pobre, erros de funcionários e falhas de respostas), tratamento injusto (situações que favorecem excessivamente a empresa) e expressão de hostilidade.

Segundo Bougie et al. (2003), a raiva é um poderoso antecedente de respostas comportamentais dos consumidores à falha do serviço, ou seja, encontra-se para além do efeito de insatisfação, como indicam Huefner e Hunt (2000). Para Kalamas, Laroche e Makdessian (2008), à medida que aumenta a intensidade da raiva e demais emoções negativas correlatas, também aumenta a propensão dos consumidores a cobrar e agir propositadamente de forma danosa à empresa. Ademais, Consumidores com raiva tornam-se mais inclinados a se engajar em Atitudes Vingativas (Bonifield \& Cole, 2007).

Dessa forma, o desejo de vingança, juntamente com a raiva, constitui importante antecedente de atitudes vingativas por parte dos consumidores, porquanto alimenta sua necessidade de causar desconforto, produzir dano, prejudicar, punir ou simplesmente pressionar a empresa a fim de restabelecer a equidade psicológica (Andrade et al., 2013; Bechwati \& Morrin, 2003; Huefner \& Hunt, 2000; Huefner et al., 2002;), ou simplesmente alivia a raiva (Zourrig et al., 2009; Bougie et al., 2003).

Seguindo essa rota emocional, Grégoire, Laufer e Tripp (2010) desenvolveram um modelo no qual as cognições estabelecidas levam à raiva, que, por sua vez, gera um Desejo de Vingança (DV) que pode influenciar na Atitude Vingativa em si ou em seus possíveis consequentes - Desejo de Afastamento (DA) e Desejo de Reconciliação (DR) -, como se verá com maior profundidade na apresentação dos construtos a seguir.

\subsection{Construtos}

\subsubsection{O desejo de vingança (DV) e as reclamações vingativas (RV)}

Bechwati e Morrin (2003) definem o Desejo de Vingança (DV) como sentimentos retaliatórios que consumidores sentem em relação à empresa, abrangendo, por exemplo, o desejo de externar algum dano à firma, tipicamente resultantes de uma experiência de compra extremamente negativa. A raiva difere aqui do Desejo de Vingança (DV), pois é mais espontânea e menos intencionalmente planejada (Bechwati \& Morrin, 2003, 2007). O Desejo de Vingança (DV) é, porém, resultante da raiva, segundo Joireman et al. (2013).

Huefner e Hunt (2000) citam como exemplos de comportamentos vingativos a criação de custos ou perdas para a empresa (com o intuito de afetá-la financeiramente), a prática de vandalismo, o roubo, o boca-a-boca negativo (pessoal ou eletrônico) e o ataque pessoal, atitudes agressivas projetadas pelos clientes para prejudicar diretamente uma empresa ou seus funcionários (Grégoire \& Fisher, 2008; Huefner \& Hunt, 2000).

Grégoire et al. (2010) subdividem as atitudes vingativas em diretas e indiretas a partir da moderação de percepção de poder do consumidor. A vingança direta pode assumir a forma de 
Reclamação Vingativa (RV) quando os clientes desabafam seu descontentamento aos empregados da linha de frente de forma a causar incomodo às operações da empresa (Grégoire \& Fisher, 2008).

Atitudes de vingança indiretas, que tomariam forma fora das fronteiras da empresa, incluem (i) boca-a-boca negativo, quando os clientes compartilham suas más experiências com amigos e parentes (Grégoire \& Fisher, 2006), e (ii) reclamação pública online, definida como o ato de usar aplicativos online para alertar o público em geral sobre o mau comportamento de uma empresa, podendo assumir formas de logomarcas, sites ou blogs para reclamação (Ward \& Ostrom, 2006). O ato de queixar-se a um público massivo inclui uma clara intenção de "pôr a empresa em apuros." Para Aristóteles (1992) a publicização faz parte de atitudes de vingança; o vingador sente necessidade de tornar públicas suas atitudes de forma a demonstrar o restabelecimento de sua equidade psicológica ou da honra perdida.

Em vista da literatura estudada, pode-se postular que o Desejo de Vingança (DV) aumenta a probabilidade de Atitudes de Vingança (direta ou indireta), a exemplo de Reclamações Vingativas (RV) como ações diretas. Elabora-se assim a primeira hipótese deste estudo (H1) - O Desejo de Vingança (DV) influencia positivamente Atitudes de Vingança (AV), como as Reclamações Vingativas.

Segundo Bechwati e Morrin (2003), quando o Desejo de Vingança (DV) é alto, muitos consumidores optam por deixar a empresa e escolhem empresas competidoras, mesmo que disponham de altos preços na intenção de manter o afastamento do perpetrador ao qual a culpa pelo dano causado Ihe foi atribuída. A escolha de "deixar a empresa" não se enquadra como uma Atitude Vingativa (AV) em si, pois pode ser interpretada como uma resposta passiva ou flight, em oposição ao fight de atitudes mais ativas tidas como vingativas (Grégoire et al., 2009; Grégoire \& Fisher, 2008; Lee et al., 2013; McCullough et al., 1998; Zourrig et al., 2009,). Essa temática será mais bem aprofundada na continuidade, a partir do construto Desejo de Afastamento (DA) apresentado a seguir.

\subsubsection{O desejo de afastamento (DA)}

McCullough et al. (1998) e Grégoire et al. (2009) definem o Desejo de Afastamento (DA) (ou de evasão) como a necessidade dos clientes de retirarem-se de quaisquer interações com as empresas. Grégoire e Fisher (2008) afirmam que sua saída do relacionamento, estimulada pelo Desejo de Afastamento (DA), não constitui uma Atitude Vingativa (AV) porque não traduz qualquer intenção de agir contra empresas causando danos e transtornos. Lee et al. (2013) observaram que o Desejo de Afastamento (DA) é uma resposta mais racional.

Para Grégoire et al. (2009), o afastamento é uma resposta resultante do ajustamento da emoção ao problema, de forma a minimizar as emoções negativas como a raiva. Assim o Desejo de Vingança (DV) e o Desejo de Afastamento (DA) são respostas diferentes. Lee et al. (2013) observaram que clientes preferem, por vezes, se afastar a se vingar, em vista dos menores custos emocionais envolvidos nessa decisão. Em contraste, nesse modelo comportamental assume-se o Desejo de Afastamento (DA) como algo que ocorre após a prática de atos vingativos. Na busca de entender o modo como se manifesta esse Desejo de Afastamento (DA) após Atos Vingativos (AV) e se é possível uma reconciliação, chegou-se à $\mathrm{H}_{2}$ - Atitudes Vingativas (AV) influenciam positivamente o Desejo de Afastamento (DA).

Compreende-se, aqui, que a consequência da prática de Atitudes Vingativas (AV) motivadas por alto grau de Desejo de Vingança (DV) é o afastamento. O consumidor pode manter sua relação com a empresa, porém, conforme a intensidade da raiva e do Desejo de Vingança (DV) aumenta, Atitudes Vingativas (AV) mais drásticas são tomadas por ele.

Segundo Grégoire et al. (2010), dependendo do contexto, os clientes não são capazes de transformar seu Desejo de Vingança (DV) em Atitudes Vingativas (AV). Mesmo assim, embora não se materializem as Atitudes Vingativas (AV) motivadas por alto grau de Desejo de Vingança (DV), pode ocorrer a consequência do Desejo de Afastamento (DA), fato que corrobora a construir a $\mathrm{H} 3$ Atitudes Vingativas (AV) como as Reclamações Vingativas (RV) que não são mediadoras entre Desejo de Vingança (DV) e Atitudes de Afastamento (AF).

Funches (2011) alerta para proposições de danos às empresas, provenientes de sentimento de raiva, que vão muito além do simples rompimento da relação comercial (afastamento), as quais são ainda mais difíceis de detectar e, por conseguinte, dão espaço para o crescimento de empresas concorrentes. Pode citar-se como exemplos de danos causados por clientes vingativos (i) a redução de gastos com a empresa, incluindo a não recompra de determinados produtos ou serviços; (ii) a mudança de localização para outra filial da empresa; e (iii) o afastamento de determinados funcionários. De forma geral, esses clientes reduzem a tolerância a erros futuros ou simplesmente ainda não encontraram alternativa de escolha no mercado. 
Estabelece-se aqui o pressuposto de que, pelos atos de retaliação e vingança gerarem atitudes de afastamento, consumidores buscam a concorrência para obter o que precisam, aqui incluído o aumento do sentimento de satisfação, estando dispostos a pagar até mesmo mais $10 \%$ do valor em outras empresas (Carvalho, Sen, Mota, \& Lima, 2010).

\subsubsection{O desejo de reconciliação (DR)}

Muitos estudos observam as correlações entre vingança e reconciliação, bem como demonstram uma baixa correlação inversa (e.g. Aquino, Tripp, \& Bies, 2006; Crossley, 2008; Joireman et al. 2013). Sabe-se que esses construtos podem ocorrer simultaneamente como atitudes retaliatórias e reparatórias (Joireman et al. 2013).

Joireman et al. (2013) estudam o Desejo de Vingança (DV) versus o Desejo de Reconciliação (DR), observando, por exemplo, que clientes, muitas vezes após engajarem-se em boca a boca negativo online, podem praticar atos de reconciliação após ações empreendidas pelas empresas. Nesses casos, os clientes não abrem mão de dar uma lição na empresa (atos vingativos), mas não querem romper seu relacionamento (Joireman et al. 2013). Joireman et al. (2013) observaram alta correlação entre o desejo de reconciliação e atitudes de reparação, considerando o desejo de reconciliação um poderoso antídoto para atitudes vingativas.

Para Aquino et al. (2006), o desejo de reconciliação refere-se à medida da disponibilidade de um consumidor para aceitar o fracasso de uma empresa, na esperança de manter seu relacionamento. Nesse caso, quando a empresa interfere apresentando bons motivos, por meio de desculpas, explicações ou compensações, clientes são encorajados a perdoá-las, dado o impacto significativo dessas ações sobre a sua percepção (Joireman et al., 2013).

Visto que tais autores observaram o desejo de reconciliação como outra face da mesma moeda da vingança, em contraste, este estudo procura observar o desejo de reconciliação como consequente de atitudes de vingança, fato que corrobora a H4 - Há relação positiva entre Atitudes de Vingança (AV) e Desejo de Reconciliação (DR).

Assim, em caso de oferta de melhores ofertas pela empresa, como preços mais baixos, promoções, principalmente para serviços com pouca concorrência, ou quando o problema se refere a um funcionário, esse desejo pode ser maior. Com base nos estudos indicados, propõe-se verificar o modelo inicial proposto conforme apresentado na Figura 1.

H3

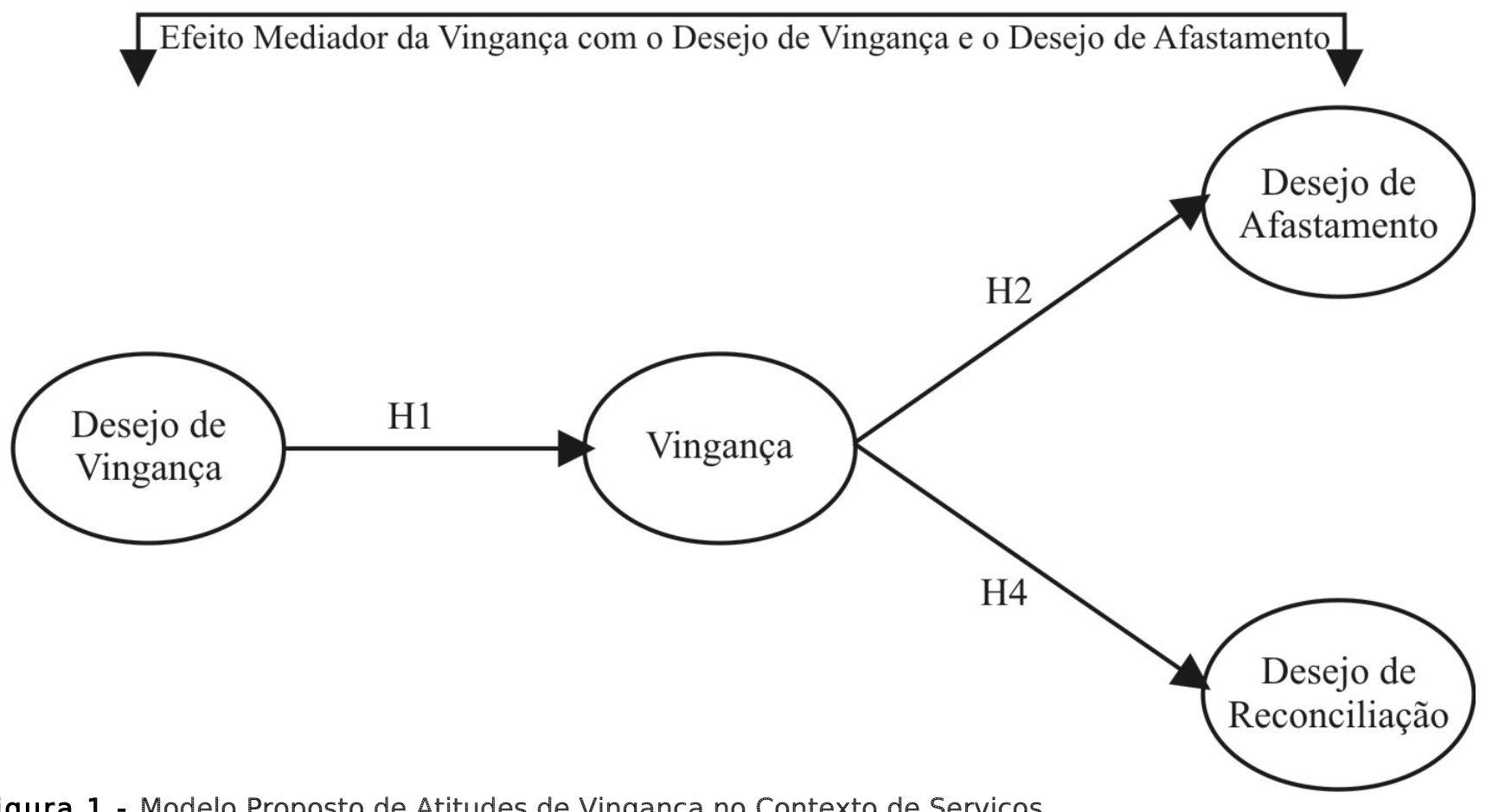

Figura 1 - Modelo Proposto de Atitudes de Vingança no Contexto de Serviços Nota. Fonte: Elaboração própria. 


\section{Procedimentos Metodológicos}

Com o intuito de analisar o comportamento de consumidores após atitudes de vingança desenvolveu-se um estudo descritivo com técnicas quantitativas. Estudos descritivos referem-se à coleta de dados em dado momento para descrever ou determinar certos traços e atributos ou relações entre variáveis (Babbie, 1999; Gil, 2002). Para o estudo descritivo, foi escolhido um tipo de levantamento, a survey interseccional, quando dados são colhidos de uma amostra em dado momento para descrever uma população maior na mesma ocasião (Babbie, 1999). A amostra deste estudo evidencia-se como não probabilística realizada por conveniência.

Para Gil (2002), por meio do questionamento às pessoas cujo comportamento se deseja conhecer tem-se, nos levantamentos, informações tratadas mediante análise quantitativa. Assim, o instrumento de coleta de dados consistiu em um questionário, cujos itens de identificação do perfil socioeconômico tratavam-se de idade, gênero, grau de instrução, renda e cidade de residência. Dividiu-se o questionário em vista dos construtos analisados, utilizando-se escalas já testadas internacionalmente, a saber: a) Atitudes Vingativas (AV) - Reclamações Vingativas (RV) (Grégoire et al., 2010), Desejo de Vingança (DV) (Joireman et al., 2013; Lee et al., 2013); Desejo de Afastamento (DA) (Grégoire et al., 2009, Lee et al., 2013) e Desejo de Reconciliação (DR) (adaptado de Joireman et al., 2013).

O filtro da pesquisa buscou identificar se os consumidores já praticaram atos com o objetivo de provocar danos à empresa ou aos funcionários após problemas em serviços, mediante análise dos itens "qual o serviço", "qual a empresa" e "há quanto tempo ocorreu". Consideraram-se na análise atos que ocorreram dentro do período de dois anos e, nesse caso, avaliaram-se apenas situações ocorridas no contexto de serviços. As escalas da pesquisa foram do tipo Likert com sete pontos, seguindo o padrão das escalas citadas. Nesse tipo de escala os respondentes expressam seu grau de concordância em relação a um conjunto de itens ou afirmações (Rodrigues \& Paulo, 2012), sendo o grau 1 = discordo totalmente e 7 = concordo totalmente.

Realizaram-se pré-testes virtuais e físicos entre os meses de janeiro e fevereiro de 2014 e a aplicação da pesquisa propriamente dita ocorreu em março de 2014. Integraram a amostra do préteste virtual 21 pesquisas e a amostra física do pré-teste 20 pesquisas. Constituíram a amostra final 219 respondentes. Os pré-testes buscaram, segundo os conceitos de Costa (2011, p. 108), verificar as validades de translação (conteúdo e face), associadas à "aferição qualitativa do conteúdo e da forma de apresentação dos itens da escala", resultando na inserção, na versão final do questionário, da expressão "punir a empresa por algo", além da realização de pequenas correções nos itens de análise.

Para a análise dos dados utilizou-se a técnica da Modelagem de Equações Estruturais (MEE), que, segundo Hair, Black, Babin, Anderson \& Tatham (2009, p. 548-549), consiste em uma técnica multivariada que permite a estimação simultânea de múltiplas equações. É apropriada para múltiplos construtos, cada um representado por diversas variáveis medidas. Tais análises foram antecedidas de testes de normalidade, homoscedasticidade e linearidade (Rodrigues \& Paulo, 2012). O software utilizado para a estimação do modelo foi o Analysis of Moment Structures - AMOS, por meio da técnica de estimação da máxima verossimilhança (maximum likelihood - ML).

A análise abrangeu a verificação dos tipos de mediação, que ocorre quando uma variável (M) exerce determinado poder sobre duas outras variáveis, uma dependente $(Y)$ e outra independente (X). Assim, enquanto o efeito direto sem uma variável mediadora seria $Y-X=c$, quando há uma variável mediadora, os efeitos diretos e indiretos são representados matematicamente da seguinte forma: $Y-M-X=c=d+a b$, em que " $c$ " é igual ao efeito total, "d" é igual ao efeito indireto intermediado pela variável mediadora, "a" é igual ao efeito entre Y e M e "b" é igual ao efeito entre M e X (Hayes, 2009). A indicação foi feita por bootstrapping de 2000 reamostras com o intervalo de confiança em $95 \%$. A seguir apresentam-se os resultados obtidos com a pesquisa e sua consequente análise.

\section{Apresentação e Análise de Dados}

Dentre os 219 respondentes pesquisados, mais de $78 \%$ têm até 25 anos, $12 \%$ entre 26 e 32 e 10\% acima de 33 anos, sendo $53 \%$ do sexo masculino e $47 \%$ do sexo feminino. O grupo compôs-se, em sua grande maioria $(61,6 \%)$, de pessoas que tiveram problemas com empresas prestadoras de serviços no período entre dois anos até um mês que antecedeu o período de aplicação do questionário, $75,3 \%$ das quais reclamaram diretamente à empresa. Desses respondentes, $62,1 \%$ ainda informaram que tiveram problemas diretamente com funcionários, 28,4\% informaram que seu problema estava relacionado a ambos (funcionário e empresa) e 9,6\% afirmaram que o problema foi relacionado exclusivamente à empresa prestadora de serviços. 
A análise do modelo proposto com base nas quatro hipóteses anteriormente indicadas iniciouse com a aplicação dos testes referentes a Análise Fatorial Exploratória (AFE). Assim, realizou-se o teste de esfericidade de Bartlett e retirou-se a medida de adequacidade da amostra Kaiser-MeyerOlkin (KMO), com todas as variáveis no modelo proposto. Os resultados indicaram a medida KMO igual a 0,782 e o qui-quadrado do teste de esfericidade de Bartlett igual a 12154,929, com significância igual a 0,00 para 990 graus de liberdade. Em suma, todos os resultados foram satisfatórios para a realização da AFE.

Na Tabela 1 apresentam-se os índices resultantes da AFE. Verificou-se se a carga fatorial de cada item no construto é de no mínimo 0,50, se o cálculo do alfa de Cronbach é de no mínimo 0,70 e se a variância extraída é de no mínimo 0,50 (Hair et al., 2009). A medida KMO das amostras foi de 0,795, o R2 acumulado de $71,5 \%$ e o coeficiente do alfa de Cronbach de 0,909.

Tabela 1:

Análise Fatorial Exploratória

\begin{tabular}{|c|c|c|c|c|c|c|c|}
\hline & $\begin{array}{l}\text { Médi } \\
\text { a }\end{array}$ & $\begin{array}{l}\text { Desvio- } \\
\text { Padrão }\end{array}$ & $\begin{array}{c}\text { Carga } \\
\text { Fatori } \\
\text { al }\end{array}$ & $\begin{array}{l}\text { Comunalid } \\
\text { ades }\end{array}$ & $\begin{array}{l}\text { Anti- } \\
\text { Image } \\
\text { m }\end{array}$ & Alfa & $\begin{array}{l}\text { Variância } \\
\text { Extraída }\end{array}$ \\
\hline $\begin{array}{l}\text { Desejo de } \\
\text { vingança }\end{array}$ & & & & & & $\begin{array}{l}0,8 \\
58\end{array}$ & 0,548 \\
\hline DV1 & 3,16 & 2,275 & 0,740 & 0,548 & $808^{a}$ & & \\
\hline DV2 & 4,41 & 2,100 & 0,804 & 0,647 & $841^{a}$ & & \\
\hline $\mathrm{DV} 3$ & 4,15 & 2,188 & 0,797 & 0,635 & $907^{-}$ & & \\
\hline DV4 & 4,60 & 2,099 & 0,843 & 0,711 & $861^{a}$ & & \\
\hline DV5 & 3,07 & 2,178 & 0,714 & 0,509 & $830^{\mathrm{a}}$ & & \\
\hline DV6 & 4,59 & 2,254 & 0,587 & 0,345 & $856^{a^{-}}$ & & \\
\hline DV7 & 5,27 & 2,167 & 0,665 & 0,442 & $911^{a}$ & & \\
\hline $\begin{array}{c}\text { Reclamaçấo } \\
\text { Vingativa }\end{array}$ & & & & & & $\begin{array}{l}0,7 \\
86\end{array}$ & 0,676 \\
\hline RVI & 1,75 & 1,977 & 0,799 & 0,638 & $857^{a}$ & & \\
\hline RV2 & 1,47 & 1,585 & 0,837 & 0,701 &, $717^{a}$ & & \\
\hline$R V 3$ & 1,17 & 1,297 & 0,895 & 0,801 & $700^{\mathrm{a}}$ & & \\
\hline RV4 & 2,03 & 2,19 & 0,750 & 0,562 & $803^{--}$ & & \\
\hline $\begin{array}{l}\text { Desejo de } \\
\text { Afastamento }\end{array}$ & & & & & & $\begin{array}{l}0,9 \\
01\end{array}$ & 0,778 \\
\hline $\mathrm{DA} 1$ & 4,84 & 2,28 & 0,89 & 0,79 & $891^{a}$ & & \\
\hline DA2 & 5,20 & 2,21 & 0,93 & 0,86 & $760^{a}$ & & \\
\hline DA3 & 5,13 & 2,26 & 0,93 & 0,86 & $757^{a-}$ & & \\
\hline DA4 & 4,40 & 2,39 & 0,77 & 0,60 & $938^{\mathrm{a}}$ & & \\
\hline $\begin{array}{l}\text { Desejo de } \\
\text { Reconciliação }\end{array}$ & & & & & & $\begin{array}{l}0,8 \\
96\end{array}$ & 0,711 \\
\hline DR1 & 3,05 & 2,15 & 0,83 & 0,69 & $886^{a}$ & & \\
\hline $\mathrm{DR} 2$ & 2,81 & 1,87 & 0,87 & 0,76 & $830^{\mathrm{a}-}$ & & \\
\hline DR3 & 2,67 & 1,81 & 0,88 & 0,77 & $846^{a}$ & & \\
\hline DR4 & 2,93 & 2,00 & 0,83 & 0,69 & $871^{a}$ & & \\
\hline DR5 & 2,60 & 1,89 & 0,80 & 0,64 & $904^{\mathrm{a}}$ & & \\
\hline
\end{tabular}

Nota. Fonte: Pesquisa Direta.

Em seguida, realizaram-se análises fatoriais confirmatórias construto a construto no intuito de verificar se os conjuntos de indicadores utilizados estavam medindo o que teoricamente deveriam medir, apresentando validade convergente e discriminante, e também se eram consistentes, apresentando unidimensionalidade, ou se seriam necessárias reespecificações.

Conforme indicações de Fornell e Larker (1981) e de Garver e Mentzer (1999), em primeiro momento foram avaliadas a confiabilidade (alfa de Cronbach) e a validade convergente (cargas fatoriais) das variáveis, advindos da Análise Fatorial Exploratória (AFE), assim como outras medidas advindas da Análise Fatorial Confirmatória (AFC), como a confiabilidade composta (CR - satisfatória quando $C R>0,7$ ), a validade convergente pela variância média extraída (AVE - satisfatória quando AVE $>0,5$ ), a variância quadrada compartilhada (MSV) e a variância quadrada média compartilhada (ASV). Para uma estimação satisfatória tem-se que CR > AVE (Fornell \& Larker, 1981; Garver \& Mentzer, 1999).

Objetivou-se, nesse momento, verificar se o conjunto de indicadores utilizados media o que deveria medir segundo a literatura, ou seja, se apresentava validade convergente e discriminante, além de analisar se apresentava unidimensionalidade. Como se observa nas Tabelas 2 e 3, todos os resultados foram satisfatórios. 
$\mathrm{Na}$ abordagem two-step approach, sugerida por Anderson e Gerbing (1988) e Schumacker e Lomax (1996), em primeiro momento observa-se o modelo de mensuração, em seguida estabelecido o modelo estrutural, ou seja, o modelo que inclui as relações entre construtos.

Estimou-se o modelo com todos os construtos validados e realizou-se sua análise por meio da especificação do modelo geral de mensuração. Observaram-se assim as medidas de ajustes estatísticos: (a) ajustes absolutos: qui-quadrado $(x 2)$ e raiz do erro quadrático médio de aproximação, considerado o badness-of-fit (Root Mean Square Error of Approximation [ RMSEA]); (b) medidas incrementais de ajustes: o índice incremental com retirada de graus de liberdade Incremental Fit Index (IFI), Nonnormed Fit Index (NNFI) ou Tucker-Lewis Coefficient (TLI); o Comparative Fit Index (CFI) - que mede quão melhor o modelo se ajusta em relação ao modelo base ou nulo; e (c) a avaliação do ajuste parcimonioso do modelo por meio do Normed Chi-Square (quiquadrado/gl ou X²/gl). Os índices estão explícitos na Tabela 4.

Tabela 2:

Propriedades das medidas escalares

\begin{tabular}{|c|c|c|}
\hline Construto & $\begin{array}{l}\text { Carga } \\
\text { Fatorial }\end{array}$ & Alfa \\
\hline Desejo de vingança & & 0,858 \\
\hline DV1 & 0,740 & \\
\hline DV2 & 0,804 & \\
\hline DV3 & 0,797 & \\
\hline DV4 & 0,843 & \\
\hline DV5 & 0,714 & \\
\hline DV6 & 0,587 & \\
\hline DV7 & 0,665 & \\
\hline Reclamação Vingativa & & 0,786 \\
\hline$R V 1$ & 0,799 & \\
\hline $\mathrm{R} V 2$ & 0,837 & \\
\hline $\mathrm{R} V \overline{3}$ & 0,895 & \\
\hline RV4 & 0,750 & \\
\hline Desejo de Afastamento & & 0,901 \\
\hline DA1 & 0,89 & \\
\hline$\overline{D A} 2$ & 0,93 & \\
\hline DA3 & 0,93 & \\
\hline DA4 & 0,77 & \\
\hline $\begin{array}{c}\text { Desejo de } \\
\text { Reconciliação }\end{array}$ & & 0,896 \\
\hline DRI & 0,83 & \\
\hline $\mathrm{DR} 2$ & 0,87 & \\
\hline DR3 & 0,88 & \\
\hline DR4 & 0,83 & \\
\hline DR5 & 0,80 & \\
\hline
\end{tabular}

Nota. Fonte: Pesquisa Direta.

Tabela 3:

Propriedades da confiabilidade e da validade convergente e discriminante

\begin{tabular}{|c|c|c|c|c|c|c|c|c|}
\hline Construtos & CR & AVE & MSV & ASV & Afastamento & $\begin{array}{c}\text { Reclamação } \\
\text { Vingativa }\end{array}$ & $\begin{array}{c}\text { Desejo } \\
\text { de } \\
\text { Vingança }\end{array}$ & Reconciliação \\
\hline Afastamento & 0,907 & 0,714 & 0,102 & 0,065 & $0,845^{*}$ & & & \\
\hline Vingança & 0,833 & 0,510 & 0,061 & 0,027 & 0,142 & $0,714^{*}$ & & \\
\hline $\begin{array}{l}\text { Desejo de } \\
\text { Vingança }\end{array}$ & 0,861 & $0,475 * *$ & 0,102 & 0,055 & 0,320 & 0,247 & $0,689^{*}$ & \\
\hline Reconciliação & 0,899 & 0,640 & 0,072 & 0,024 & $-0,268$ & $-0,021$ & $-0,012$ & $0,800 *$ \\
\hline
\end{tabular}

Nota. Fonte: Pesquisa Direta.

Indicações satisfatórias segundo Fornell e Larker (1981) e Garver e Mentzer (1999): CR > 0,7; AVE > 0,5; CR > AVE; MSV < AVE e ASV < AVE. *Correlações satisfatórias segundo Tabachnick e Fidell $(2001)<0,85$. **Próximo ao limite, porém, dentro dos valores aceitáveis.

Tabela 4:

Índices de ajustamento estatístico - modelo de mensuração

$\begin{array}{cccccccc}\begin{array}{c}\text { qui- } \\ \text { uadrado } \\ (\chi 2)\end{array} & \begin{array}{c}\text { Graus de } \\ \text { Liberdade } \\ \text { (gl) }\end{array} & \begin{array}{c}\text { quadrado/gl } \\ \text { quadra } \\ \text { ou X2/gl }\end{array} & \text { p } & \text { RMSEA } & \text { IFI } & \text { TLI } & \text { CFI } \\ 317,792 & 179 & 1,775 & 0,000 & 0,060 & 0,945 & 0,935 & 0,944\end{array}$

Nota. Fonte: Pesquisa Direta. 
De acordo com as indicações de Tabachnick e Fidell (2001) os seguintes índices são satisfatórios: x2/gl < 3; IFI, TLI e CFI > 0,90; e RMSEA $<0,08$.

Os índices de ajustamento do modelo estrutural foram (Tabela 5):

Tabela 5:

\begin{tabular}{cccccccc} 
Índices de ajustamento estatístico & - modelo estrutural \\
qui- & $\begin{array}{c}\text { Graus de } \\
\text { quadrado } \\
\text { Liberdade } \\
(\chi 2)\end{array}$ & $\begin{array}{c}\text { qui- } \\
\text { (gl) }\end{array}$ & $\begin{array}{c}\text { quadrado/gl } \\
\text { ou X } \mathbf{X}^{2} / \mathrm{gl}\end{array}$ & RMSEA & IFI & TLI & CFI \\
\hline 348,740 & 182 & 1,916 & 0,000 & 0,065 & 0,934 & 0,923 & 0,933
\end{tabular}

Nota. Fonte: Pesquisa Direta.

De acordo com as indicações de Tabachnick e Fidell (2001) os seguintes índices são satisfatórios: $\chi 2 / g \mid<3$; |Fl, TLI e CFI > 0,90; e RMSEA $<0,08$.

Realizou-se, então, o teste de hipóteses a partir da avaliação da significância dos parâmetros padronizados. De acordo com os resultados da Tabela 6 , inicialmente uma hipótese foi rejeitada, pois, como se pode evidenciar, o valor $t$ não foi estatisticamente significante (menor que 1,96 ) e o valor de $p$ foi maior que 0,05 .

Tabela 6:

Testes de hipóteses do modelo estrutural

\begin{tabular}{|c|c|c|c|c|c|c|c|}
\hline $\begin{array}{c}\text { Variável } \\
\text { Dependente }\end{array}$ & $\begin{array}{c}\text { Variável } \\
\text { Independente }\end{array}$ & $\begin{array}{c}\text { Cargas } \\
\text { Fatoriais não- } \\
\text { padronizadas } \\
\text { (b) }\end{array}$ & $\begin{array}{l}\text { Erros- } \\
\text { padrão } \\
(\varepsilon)\end{array}$ & $\begin{array}{c}\text { Cargas } \\
\text { Fatoriais } \\
\text { padronizadas } \\
(ß)\end{array}$ & $\begin{array}{c}\text { Teste } \\
\mathrm{t}\end{array}$ & $P$ & Hipóteses \\
\hline Vingança & $\begin{array}{l}\text { Desejo de } \\
\text { Vingança }\end{array}$ & 0,329 & 0,080 & 0,238 & 2,996 & 0,003 & H1 Aceita \\
\hline $\begin{array}{c}\text { Desejo de } \\
\text { Afastamento }\end{array}$ & Vingança & 0,213 & 0,100 & 0,156 & 2,117 & 0,034 & H2 Aceita \\
\hline $\begin{array}{c}\text { Desejo de } \\
\text { Reconciliação }\end{array}$ & Vingança & $-0,022$ & 0,090 & $-0,018$ & $-0,239$ & 0,811 & $\begin{array}{c}\mathrm{H} 4 \\
\text { Rejeitada }\end{array}$ \\
\hline
\end{tabular}

Nota. Fonte: Pesquisa Direta.

A aceitação da H1: O Desejo de Vingança (DV) influencia positivamente Atitudes de Vingança (AV) como as Reclamações Vingativas reforçam a necessidade de consumidores na relação de troca malsucedida causarem dano, prejuízo, desconforto, punição, ou simplesmente pressionarem a empresa a fim de restabelecer a equidade psicológica (Andrade et al., 2013; Bechwati \& Morrin, 2003; Huefner \& Hunt, 2000; Huefner et al., 2002).

A aceitação da H2: Atitudes Vingativas (AV) influenciam positivamente o Desejo de Afastamento (DA) e corrobora a necessidade dos clientes de, após adotarem Atitudes Vingativas (AV) motivadas por um alto grau de Desejo de Vingança (DV), deixarem de interagir com a empresa que julgam culpada pela experiência malsucedida (Grégoire, Laufner, \& Tripp, 2009; McCullough et al., 1998).

A não aceitação da H4: Há relação positiva entre Atitudes de Vingança (AV) e Desejo de Reconciliação (DR) e isso conduz a uma reflexão acerca da tolerância de consumidores brasileiros em relação ao fracasso de uma empresa em satisfazer seus clientes que deve ser aprofundada em estudos futuros. Joireman et al. (2013) apontam que por meio da interferência da empresa em pedir desculpas e/ou dar explicações e compensações, faz com que os clientes possam ficar encorajados a perdoá-las, uma vez que há impacto direto e significativo dessas ações sobre suas percepções.

Para se verificar a H3: Atitudes Vingativas (AV) como as Reclamações Vingativas (RV) não são mediadoras entre Desejo de Vingança (DV) e Atitudes de Afastamento (AF). O próximo passo consistiu em avaliar o tipo de mediação utilizada a partir da realização de um teste para verificação dos efeitos diretos e indiretos na mediação do construto Desejo de Vingança e Afastamento.

Na primeira técnica, iniciou-se a verificação do efeito direto e do nível de significância dessa relação sem as variáveis mediadoras no modelo estrutural. O segundo passo consistiu na inclusão da relação mediadora entre as variáveis no modelo estrutural e na indicação do bootstrapping de 2000 reamostras com o intervalo de confiança em $95 \%$. A técnica para verificar o intervalo de confiança desse efeito mediador foi o bias-corrected method. Essa técnica consiste em fazer com que a distribuição não tenha viés de problemas com normalidade na construção de estimadores. Seguem os resultados conforme Tabela 7. 
Pode-se assegurar, desse modo, que os clientes motivados por alto Desejo de Vingança (DV), mesmo ao não materializarem Atitudes Vingativas (AV), mantêm seu Desejo de Afastamento (DA) da empresa perpetradora do dano (Grégoire et al., 2010).

Em seguida, utilizou-se a técnica de Preacher e Hayes $(2004,2008)$ e Preacher, Rucker e Hayes (2007) para a verificação do efeito de mediação na hipótese H3, indicada com o Desejo de Afastamento como a variável dependente (VD), o Desejo de Vingança como a variável independente (VI) e a Vingança como variável mediadora (Med). Assim, para a amostra dos respondentes, o efeito direto (B) do Desejo de Vingança junto ao Desejo de Afastamento foi igual a 0,3528, obtendo $t=4,3218$ e $p<0,000$ e $F=18,6776$ com $p=0,000$.

Tabela 7:

Análise dos tipos de mediação no modelo estrutural

\begin{tabular}{|c|c|c|c|c|c|}
\hline Variáveis & $\begin{array}{l}\text { Efeitos } \\
\text { Diretos } \\
\text { sem } \\
\text { Mediadora } \\
\text { (sig.) }\end{array}$ & $\begin{array}{c}\text { Efeitos } \\
\text { Diretos } \\
\text { com } \\
\text { Mediadora } \\
\text { (sig.) }\end{array}$ & $\begin{array}{l}\text { Efeitos } \\
\text { Indiretos }\end{array}$ & $\begin{array}{c}\text { Tipo de } \\
\text { Mediação }\end{array}$ & Hipótese \\
\hline $\begin{array}{l}\text { VD = Afastamento } \\
\text { VI = Desejo de } \\
\text { Vingança } \\
\text { Med = Vingança }\end{array}$ & $\begin{array}{c}0,433 \\
(* * *)\end{array}$ & $\begin{array}{c}0,300 \\
(0,013)\end{array}$ & $\begin{array}{c}0,017 \\
(0,379)\end{array}$ & $\begin{array}{c}\text { Sem } \\
\text { Mediação }\end{array}$ & $\begin{array}{c}\text { H3 } \\
\text { Aceita }\end{array}$ \\
\hline
\end{tabular}

Nota. Fonte: Pesquisa Direta.

O próximo passo consistiu em incluir as variáveis mediadoras no modelo juntamente com a variável independente, no intuito de verificar o efeito (B) do coeficiente. Assim, apresenta-se a Tabela 8 , contemplando os efeitos com todas as variáveis no modelo.

Tabela 8:

Efeito de mediação da vingança entre o desejo de vingança e o desejo de afastamento

\begin{tabular}{|c|c|c|c|c|}
\hline Variáveis & Efeito (ß) & Erro padrão & $\mathrm{T}$ & $p$ \\
\hline $\begin{array}{l}\text { Vingança } \rightarrow \text { Desejo de } \\
\text { Afastamento }\end{array}$ & 0,1091 & 0,0876 & 1,2454 & 0,2143 \\
\hline $\begin{array}{l}\text { Desejo de Vingança } \rightarrow \\
\text { Desejo de Afastamento }\end{array}$ & 0,3276 & 0,0840 & 3,8998 & 0,0001 \\
\hline
\end{tabular}

Nota. Fonte: Pesquisa Direta (2014).

O efeito (B) tende a ficar mais fraco ou insignificante quando indicado com todas as variáveis mediadoras no modelo. Assim, percebe-se que houve fraca redução entre o Desejo de Vingança e o Desejo de Afastamento (Desejo de Afastamento $\leftarrow$ Desejo de Vingança, passando de 0,3528 para $0,3276)$.

Por último, para se verificar a significância dos efeitos indiretos na mediação, avaliou-se o viés corrigido e acelerado do intervalo de confiança a 95\% com bootstrapping com 10.000 redistribuições amostrais. Para Preacher e Hayes (2004, 2008) e Preacher et al. (2007), caso o zero esteja contido em quaisquer dos intervalos de confiança dos efeitos indiretos, há perda de significância no efeito. A Tabela 9, a seguir, indica as relações, os efeitos e os vieses corrigidos e acelerados dos baixos e altos intervalos de confiança a $95 \%$. Percebe-se que há valor zero entre os intervalos de confiança (IC), confirmando o efeito de não mediação da hipótese H3.

Tabela 9:

Análise dos efeitos corrigido e acelerado dos baixos e altos efeitos dos intervalos de confiança da mediação

Nota. Fonte: Pesquisa Direta.

\begin{tabular}{cccc}
$\begin{array}{c}\text { Relação do Efeito } \\
\text { Indireto }\end{array}$ & Efeito & Baixo IC & Alto IC \\
\hline $\begin{array}{c}\text { Desejo de Afastamento } \\
\leftarrow \text { Desejo de Vingança }\end{array}$ & 0,0252 & $-0,0115$ & 0,0804 \\
\hline
\end{tabular}

Em vista desses resultados apresentam-se as seguintes considerações finais. 


\section{Conclusões e Recomendações}

O presente estudo buscou contribuir com a ampliação do escopo de compreensão da vingança no comportamento do consumidor conforme lacuna apontada por Bechwati \& Morrin (2003), Cronin (2003), Funches et al. (2009), trazendo à luz da ciência situações de contato direto entre consumidor e empresa no contexto de prestação de serviços, bem como para o aprofundamento do estudo sobre os fatores que compõem esse fenômeno e seus consequentes (Albuquerque et al., 2011; Andrade et al., 2010; Andrade \& Brandão, 2012; Andrade et al., 2013; Fernandes \& Santos, 2008; Giglio \& Chauvel, 2002; Santos \& Fernandes, 2010).

O objetivo geral desse estudo foi, portanto, analisar os consequentes de Atitudes Vingativas de Consumidores no contexto de serviços. Pode-se apontar como principal resultado a relação de causalidade entre desejo de vingança e a prática de um tipo de ato tido como vingativo (H1), achado que reforça as descobertas de Bechwati e Morrin (2003) ao definirem o Desejo de Vingança (DV) como sentimento retaliatório de consumidores em relação à empresa, como o desejo de externar algum dano à firma, tipicamente resultado de uma experiência de compra extremamente negativa.

A aceitação da $\mathrm{H} 2$ confirma a relação também positiva entre a vingança e o desejo de afastamento. A partir dessa descoberta pode-se compreender o afastamento pós-Atitude Vingativa como uma resposta decorrente no ajustamento da emoção ao problema, de forma a minimizar as emoções negativas como a raiva (Grégoire et al., 2009).

Além disso, observou-se que não há relação de mediação entre o desejo de vingança e o desejo de afastamento (H3), o que fortalece a relação entre esses construtos. Lee et al. (2013) observaram que clientes por vezes preferem se afastar a se vingar em vista dos menores custos emocionais. A escolha de "deixar a empresa" não é definida como uma Atitude Vingativa (AV) em si, pois pode ser interpretada como uma resposta passiva ou flight, em oposição ao fight de atitudes mais ativas tidas como vingativas (Grégoire \& Fisher, 2008; Grégoire et al., 2009; Lee et al., 2013; McCullough et al., 1998; Zourrig et al., 2009).

No presente estudo não se evidenciou relação significativa entre a vingança e o desejo de reconciliação (H4), fato que conduz a uma reflexão acerca da tolerância de consumidores brasileiros em relação ao insucesso em satisfazer os clientes de uma empresa que deve ser aprofundada em estudos futuros. (e.g. estudos transculturais). Estudo futuros podem ser examinados com a inclusão de efeitos moderadores provenientes de Joireman et al. (2013) com relação às atitudes dos consumidores acerca de desculpas, explicações e compensações provenientes das empresas aos seus clientes.

Dessa forma, tem-se que consumidores de serviços pesquisados em um ambiente real de compras, após envolverem-se em desentendimentos que estimulam o desejo de vingança contra prestadores de serviços, objetivam não mais voltar a consumir ou a se relacionar com a empresa promotora de danos.

Ao se relacionar a ausência de mediação entre o desejo de vingança e o desejo de afastamento, pode-se concluir que, no ambiente estudado, basta haver o desejo de vingança para que consumidores se afastem das empresas prestadoras do serviço avaliado. A não significância entre a vingança e a reconciliação afirma a importância da prestação de serviços com qualidade e da atenção ao gerenciamento do pós-venda, antecipando desejos de vingança latentes em consumidores, visto que é remota a possibilidade de reconciliação. Assim, como implicações gerenciais, observa-se a necessidade de prestadores de serviços atentarem aos clientes tidos muitas vezes como indesejáveis por danos que possam ter causado. Isso porque, além da perda desses clientes, que não retornarão à relação, os prestadores de serviços podem ainda ter que amargar os efeitos de boca a boca negativo, transmitidos de forma amplificada pelas suas redes pessoais em vista da maior possibilidade de alcance virtual. Vale ressaltar que embora os custos emocionais não tenham sido tratados neste trabalho, são também apontados como relevantes pela literatura, estando associados ao desejo de afastamento. Ações corretivas evidenciam-se, assim, inclusive como ações tidas como responsáveis no trato com o consumidor.

No âmbito das implicações acadêmicas, em contraste com estudos internacionais que apontam a existência dos construtos desejo de afastamento e desejo de reconciliação como alternativos às atitudes vingativas, este estudo apresentou consequências de atitudes vingativas de forma a evidenciar novas complexidades em aspectos negativos no comportamento do consumidor.

Como limitações desta pesquisa, não foi possível resgatar construtos pertinentes a esse contexto, como raiva, percepção de justiça, tolerância, insatisfação, ou mesmo adentrar o universo de outras atitudes tidas como vingativas, como campanhas virtuais, ataques aos empregados das empresas de serviços, dentre outras. Além disso, não foi possível evidenciar efeitos considerados como moderadores. É importante que se avaliem ainda tais resultados nos diferentes tipos de 
serviços ou culturas, posto que, em vista das diversas naturezas, é possível que peculiaridades contratuais ou a falta de concorrência interfiram no modelo, resultando em diferenças significativas nas análises finais.

Indica-se, portanto, para pesquisas futuras, a inserção do relacionamento de novos construtos no modelo proposto de atitudes vingativas. Sugere-se, ainda, o estudo de emoções como o medo de vingança e atitudes de arrependimento, bem como de efeitos moderadores como a personalidade e o tempo, e de mediadores como ações reparadoras das empresas. Estes pontos surgiram em estudos qualitativos prévios, mas não puderam ser aprofundados nesta análise. Recomenda-se, do mesmo modo, a aplicação de estudos comparativos entre o ambiente brasileiro e outros países, de forma a detectar peculiaridades no cenário local que promovam estratégias específicas.

\section{Referências}

Albuquerque, F. M. F., Pereira, R. C. F., \& Bellini, C. G. P. (2011). Do repertório de comportamentos do consumidor: retaliação e vingança no ciberespaço. Revista de Administração, 46(2), 135-149.

Anderson, J. C., \& Gerbing, D. W. (1988). Structural equation modeling in practice: a review and recommended two-step approach. Psychological Bulletin, 103(3), 411-423.

Andrade, D. M., Balassiano, M., \& Brandão, M. M. (2013). Modelo estrutural do comportamento de retaliação e vingança do consumidor insatisfeito. Revista Brasileira de Marketing, 12(1), 51 -72.

Andrade, D. M., \& Brandão, M. M. (2012). Boca a Boca Eletrônico como Forma de Retaliação: uma Aplicação de Modelagem de Equações Estruturais para Determinar o Comportamento PósReclamação de Consumidores Insatisfeitos-Ofendidos. Revista ADM.MADE, 16(1), 24-44.

Andrade, D. M., Brandão, M. M., Pereira, S. J. N., \& Cunha, E. S. (2010). Clicando e Reclamando: Uma Análise de Consumidores Insatisfeitos com Compras Online. Anais do Semead, São Paulo, SP, Brasil, 18.

Aquino, K., Tripp, T., \& Bies, R. (2006). Getting Even or Moving on? Power, Procedural Justice, and Types of Offense as Predictors of Revenge, Forgiveness, Reconciliation, and Avoidance in Organizations. Journal of Applied Psychology, 91(3), 653-658.

Aristóteles. (1992). Ética a Nicomacos. Brasília: Edunb.

Babbie, E. (1999). Métodos de Pesquisa de Survey. Belo Horizonte: Editora da UFMG.

Bechwati, N. N., \& Morrin, M. (2003). Outraged Consumers: Getting Even at the Expense of Getting a Good Deal. Journal of Consumer Psychology, 13(4), 44-53.

Bechwati, N. N., \& Morrin, M. (2007). Understanding Voter Vengeance. Journal of Consumer Psychology, 17(4), 277-291.

Bonifield, C., \& Cole, C. (2007). Affective responses to service failure: Anger, regret, and retaliatory versus conciliatory responses. Marketing Letters, 18(1-2), 85-99.

Bougie, R., Pieters, R., \& Zeelenberg, M. (2003). Angry Customers Don't Come Back, They Get Back: The Experience and Behavioral Implications of Anger and Dissatisfaction in Services. Journal of the Academy of Marketing Science, 31(4), 377-393.

Carvalho, S. W., Sen, S., Mota, M. O., \& Lima, R. C. (2010). Consumer Reactions to CSR: A Brazilian Perspective. Journal of Business Ethics, 91(2), 291-310.

Costa, F. J. (2011). Mensuração e Desenvolvimento de Escalas: Aplicações em Administração. Rio de Janeiro: Editora Ciência Moderna.

Cronin Jr., J. J. (2003). Looking back to see forward in services marketing some ideas to consider. Managing Service Quality, 13(5), 332-337.

Crossley, C. D. (2008). Emotional and Behavioral Reactions to Social Undermining: A Closer Look at Perceived Offender Motives. Organizational Behavior and Human Decision Processes, 108(1), 14-24.

Fernandes, D., \& Santos, C. (2008). As Consequências Comportamentais da Insatisfação dos Clientes. Revista de Administração Contemporânea, 12(Edição Especial), 147-174.

Fornell, C, \& Larker, D. (1981). Evaluating structural equation models with unobservable variables and measurement error. Journal of Marketing Research, 18(1), 39-50.

Funches, V. (2011). The consumer anger phenomena: causes and consequences. Journal of Services Marketing, 25(6), 420-428. 
Funches, V., Markley, M., \& Davis, L. (2009). Reprisal, retribution \& requital: Investigating customer retaliation. Journal of Business Research, 62(2), 231-239.

Garver, M. S., \& Mentzer, J. T. (1999). Logistics research methods: employing structural equation modeling to test for construct validity. Journal of Business Logistics, 20(1), 33-57.

Giglio, E. A. M., \& Chauvel, M. A. (2002). Reclamação e cultura brasileira: um estudo baseado na análise de cartas de consumidores insatisfeitos à imprensa. Anais do Encontro Nacional da Associação Nacional de Pós-Graduação e Pesquisa em Administração, Curitiba, PR, Brasil, 26.

Gil, A. C. (2002). Como Elaborar Projetos de Pesquisa. São Paulo: Atlas.

Grégoire, Y., \& Fisher, R. (2008). Customer Betrayal and Retaliation: When Your Best Customers Become Your Enemies. Journal of the Academy of Marketing Science, 36(2), 247-261.

Grégoire, Y., Laufer, D., \& Tripp, T. (2010). A Comprehensive Model of Customer Direct and Indirect Revenge: understanding the Effects of Perceived Greed and Customer Power. Journal of the Academy of Marketing Science, 38(6), 738-758.

Grégoire, Y., Laufer, D., Tripp, T., \& Legoux, R. (2009). When Customer Love Turns into Lasting Hate: The Effects of Relationship Strength and Time on Customer Revenge and Avoidance. Journal of Marketing, 73(6), 18-32.

Hair, J. F.; Black, W. C.; Babin, B. J.; Anderson, R. E., \& Tatham, R. L. (2009). Análise Multivariada de Dados (6 ed.). Porto Alegre: Bookman.

Hayes, Andrew F. (2009). Beyond Baron and Kenny: Statistical Mediation Analysis in the New Millennium. Communication Monographs, 76(4), 408-420.

Hopkins, L. (2013). Revenge or Reconciliation? A Rejection-Based Model of Firm-Induced Relationship Termination. Tese de doutorado, Kennesaw State University, Kennesaw, United States.

Huefner, J. C., \& Hunt, H. K. (2000). Consumer retaliation as a response to dissatisfaction. Journal of Consumer Satisfaction, Dissatisfaction \& Complaining Behavior, 13, 61-82.

Huefner, J. C., Hunt, H. K., Parry, L. B., Payne, C. R., Otto, S. D., Huff, S. C., \& Swenson, M. J. (2002). Consumer retaliation: confirmation and extension. Journal of Consumer Satisfaction, Dissatisfaction \& Complaining Behavior, 15, 114-127.

Joireman, J., Grégoire, Y., Devezer, B., \& Tripp, T. (2013). When do customers offer firms a "second chance" following a double deviation? The impact of inferred firm motives on customer revenge and reconciliation. Journal of Retailing, 89(3), 315-337.

Kalamas, M., Laroche, M., \& Makdessian, L. (2008). Reaching the boiling point: Consumers' negative affective reactions to firm-attributed service failures. Journal of Business Research, 61(8), 813-824.

Lee, Jin-Soo, Pan, S., \& Tsai, H. (2013). Examining perceived betrayal, desire for revenge and avoidance, and the moderating effect of relational benefits. International Journal of Hospitality Management, 32, 80-90.

McCullough, M. E., Rachal, K. C., Sandage, S. J., Worthington, E. L., Brown, S. W., \& Hight, T. L. (1998). Interpersonal forgiving in close relationships. II: Theoretical elaboration and measurement. Journal of Personality and Social Psychology, 75(6), 1586-1603.

Preacher, K. J., \& Hayes, A. F. (2004). SPSS and SAS Procedures for Estimating Indirect Effects in Simple Mediation Models. Behavior Research Methods, Instruments, and Computers, 36(4), 717-731.

Preacher, K. J., \& Hayes, A. F. (2008). Asymptotic and Resampling Strategies for Assessing and Comparing Indirect Effects in Multiple Mediator Models. Behavior Research Methods, 40(3), 879-891.

Preacher, K. J., Rucker, D. D., \& Hayes, A. F. (2007). Addressing Moderated Mediation Hypotheses: Theory, Methods, and Prescriptions. Multivariate Behavioral Research, 42(1), 185-227.

Rodrigues, A., \& Paulo, E. (2012). Introdução à Análise Multivariada. In L. J. Corrar, E. Paulo, \&J. M. Dias Filho (Orgs.). Análise Multivariada para cursos de Administração, Ciências Contábeis e Economia (pp. 1-72). São Paulo: Atlas.

Santos, C. P., \& Fernandes, D. (2010). A construção de uma tipologia dos consumidores para resposta à insatisfação. Revista de Administração e Contabilidade da Unisinos, 7(1), 25-41.

Schumacker, R. E., \& Lomax, R. G. (1996). A beginner's guide to structural equation modeling. New Jersey: Lawrence Erlbaum Associates.

Stuckless, N., Ford, D. B., \& Vitelli, R. (1995). Vengeance, anger and irrational beliefs in inmates: a caveat regarding social Desirability. Personality and Individual Differences, 18(1), 1-6. 
Stuckless, N., \& Goranson, R. (1992). The Vengeance Scale: Development of a Measure of Attitudes Toward Revenge. Journal of Social Behavior and Personality, 7(1), 25-42.

Tabachnick, B. G., \& Fidell, L. S. (2001). Using Multivariate Statistics (4th edition). Needham Heights: Allyn and Bacon.

Thelen, S., \& Shapiro, T. (2012). Predicting negative consumer reactions to services offshoring. Journal of Services Marketing, 26(3), 181-193.

Ward, J. C., \& Ostrom, A. L. (2006). Complaining to the masses: the role of protest framing in customer-created complaint web sites. Journal of Consumer Research, 33(2), 220-230.

Zourrig, H., Chebat, J., \& Toffoli, R. (2009). Consumer revenge behavior: a cross-cultural perspective. Journal of Business Research, 62(2), 995-1001. 